\title{
Uveomeningoencephalitic Syndrome
}

National Cancer Institute

\section{Source}

National Cancer Institute. Uveomeningoencephalitic Syndrome. NCI Thesaurus. Code C85218.

A systemic inflammatory process affecting the inner ear, eyes, skin, and meninges. It is manifested with hearing disorders, panuveitis, alopecia, poliosis, vitiligo, neck stiffness, headaches, and focal neurologic signs. 